\title{
Solitary Metastasis to the Facial/Vestibulocochlear Nerve Complex Case Report and Review of the Literature
}

Shafie Ariai $\mathbf{M}^{1}$, Scott D Eggers ${ }^{2}$, Caterina Giannini ${ }^{3}$, Colin L W Driscoll ${ }^{4}$ and Michael $\mathbf{J ~ L i n k}^{1 *}$

${ }^{1}$ Department of Neurologic Surgery, Mayo Clinic, Rochester, Minnesota, USA

${ }^{2}$ Department of Neurology, Mayo Clinic, Rochester, Minnesota, USA

${ }^{3}$ Department of Pathology, Mayo Clinic, Rochester, Minnesota, USA

${ }^{4}$ Department of Otorhinolaryngology, Mayo Clinic, Rochester, Minnesota, USA

\section{Abstract}

Background and Importance: Distant metastasis of mucinous adenocarcinoma from the gastrointestinal tract, ovaries, pancreas, lungs, breast or urogenital system is a well described entity. Mucinous adenocarcinomas from different primary sites are histologically identical with gland cells producing a copious amount of mucin. This report describes a very rare solitary metastasis of a mucinous adenocarcinoma of unknown origin to the facial/ vestibulocochlear nerve complex in the cerebellopontine angle.

Clinical Presentation: A 71-year-old woman presented with several month history of progressive neurologic decline and a negative extensive workup performed elsewhere. She presented to our institution with complete left facial weakness, left-sided deafness, gait unsteadiness, headache and anorexia. A repeat MRI scan of the head revealed a cystic, enhancing abnormality involving the left cerebellopontine angle and internal auditory canal. A left retrosigmoid craniotomy was performed and the lesion was completely resected. The final pathology was a mucinous adenocarcinoma of indeterminate origin. Postoperatively, the patient continued with her preoperative deficits and subsequently died of her systemic disease six weeks after discharge.

Conclusion: The facial/vestibulocochlear nerve complex is an unusual location for metastatic disease in the central nervous system. Clinicians should consider metastatic tumor as the possible etiology of an unusual appearing mass in this location causing profound neurologic deficits. The prognosis following metastatic mucinous adenocarcinoma to the cranial nerves in the cerebellopontine angle may be poor.

Keywords: Mucinous adenocarcinoma; Metastatic disease; Arachnoid

\section{Clinical Presentation}

A 71-year-old woman with an unremarkable past medical history presented in December 2012, with progressive dizziness, unsteadiness of gait and occasional falls. She also experienced diffuse bilateral frontotemporal headaches without jaw claudication or visual loss. An initial thorough evaluation was undertaken at another institution. A brain MRI with gadolinium in January 2013, showed mild generalized cerebral atrophy, minor changes of chronic and small vessel ischemia, normal cranial nerves, and no abnormal enhancement (Figure 1A). A cervical spine MRI revealed only mild spondylosis with normal signal in the spinal cord. Serum laboratory analysis was normal including complete blood count and erythrocyte sedimentation rate. In January 2013, a preliminary diagnosis of temporal arteritis was made and she was started on prednisone despite a negative temporal artery biopsy. Despite treatment with corticosteroids, her symptoms progressed such that she became bed bound.

In February 2013, she began to experience left-sided facial weakness that progressed to complete left facial paralysis over 2 weeks' time. A repeat MRI scan of the head in April 2013 was unremarkable for any new findings (Figure 1B).

The patient had no clinical improvement and prednisone was tapered and discontinued by May 2013. Throughout June 2013, the patient presented with increasing left-side hearing loss to the point of unilateral deafness.

An MRA of the head and neck in July 2013 showed only a mild narrowing at the origin of the left external carotid artery and no significant intracranial stenoses or aneurysm. A lumbar puncture with CSF analysis was remarkable for opening pressure of 166 millimeters of water (reference 100-200 mm of water), total nucleated cell count of 53 cells/dl (reference $0-5$ cells/dl), 46\% lymphocytes, $54 \%$ monocytes/ macrophages and a red cell count of 192 (reference $0-5$ cells/dl). The protein count was significantly elevated at $400 \mathrm{mg} / \mathrm{dl}$ (reference $0-35$

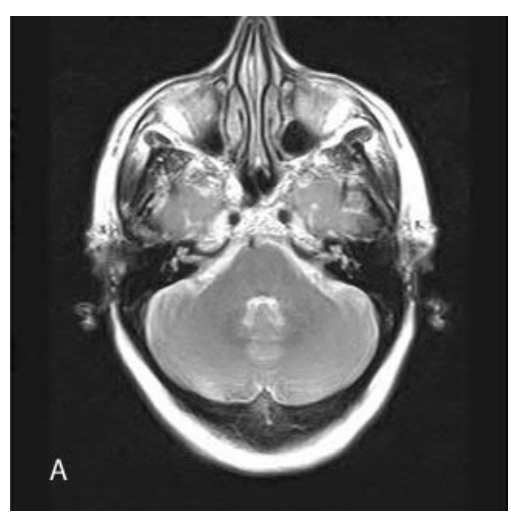

Figure 1A: Axial T2- weighted fast spin echo imaging shows no evidence for a mass lesion within the cerebellopontine region.

*Corresponding author: Link MJ, MD, Department of Neurosurgery, Mayo Clinic Rochester, Minnesota, USA, Tel: 507-284-4871; Fax: 507-284-5206; E-mail: link.michael@mayo.edu

Received January 17, 2015; Accepted February 19, 2015; Published February 27,2015

Citation: Ariai SM, Eggers SD, Giannini C, Driscoll CLW, Link MJ (2015) Solitary Metastasis to the Facial/Vestibulocochlear Nerve Complex Case Report and Review of the Literature. Surgery Curr Res 5: 218. doi:10.4172/2161-1076.1000218

Copyright: ( $) 2015$ Ariai SM, et al. This is an open-access article distributed under the terms of the Creative Commons Attribution License, which permits unrestricted use, distribution, and reproduction in any medium, provided the original author and source are credited. 


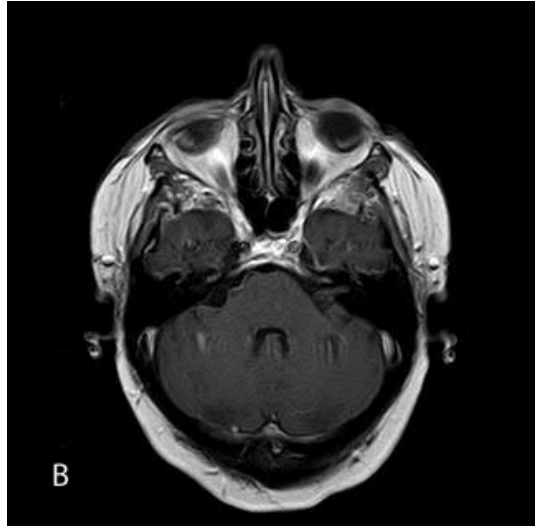

Figure 1B: Axial T1-weighted imaging with gadolinium is unremarkable for a tumor enhancement in the posterior fossa.

$\mathrm{mg} / \mathrm{dl})$. Glucose was $49 \mathrm{mg} / \mathrm{dl}$. Microbiology testing includes negative bacterial cultures and negative VZV, Lyme and HIV PCR. Serum laboratory analysis was normal or negative including paraneoplastic panel and serum inflammatory markers were negative. Angiotensin converting enzyme (ACE) level was mildly elevated at $84 \mathrm{U} / 1$ (reference $<4 \mathrm{U} / \mathrm{I})$. A repeat temporal artery biopsy was subsequently negative in July 2013.

The patient experienced severe diffuse headaches and vomited several times. She collapsed and had a generalized seizure and was transferred to our institution at the end of July 2013.

Upon transfer to our institution, the patient showed complete left facial weakness, severe left hearing loss, right beating nystagmus with visual fixation removed, abnormal leftward head impulse test consistent with left peripheral vestibulopathy. There were patchy equivocal facial sensory asymmetries, and slow cautious narrow-based gait.

A repeat MRI head was performed, now demonstrating a $1.2 \mathrm{~cm}$ enhancing cystic and solid lesion in the left cerebellopontine angle (CPA) extending into the left internal auditory canal (IAC) involving cranial nerves VII and VIII (Figure 2). The above findings, with an elevated ACE, were suspicious for sarcoidosis. A Chest CT scan was obtained which was normal, specifically without any evidence of pulmonary sarcoidosis. The patient underwent a conjunctival biopsy to further investigate for sarcoidosis which was negative. Repeat CSF studies showed normal cell count. The protein count was $812 \mathrm{mg} / \mathrm{dl}$, and glucose was $40 \mathrm{mg} / \mathrm{dl}$. Cytology as well as paraneoplastic work up was negative. CSF ACE was $4 \mathrm{mg} / \mathrm{dl}$. AFB smear, gram stain, bacterial, fungal, and mycotic cultures were unrevealing. PCR for Toxo, CMV, Lyme, HHV-6, EBV, HSV, MTB were all negative. PET scan demonstrated mild increased FDG uptake in the right lung, middle fissure, as well as possible increased uptake in the right hilar lymph nodes, and gastro-esophageal junction. The lesions were felt to be very small, have low uptake on PET and likely inflammatory rather than neoplastic as well as too small to allow for safe biopsy. Neurosurgery was then consulted for biopsy of the left CPA lesion extending into the IAC.

The patient was taken to the operative theater on August 2, 2013. A left retrosigmoid craniotomy was performed and the CPA was explored. The arachnoid of the CPA was noted to be extremely thickened and opaque. The thickened arachnoid was opened around the seventheighth cranial nerve complex, and the cyst formation seen on the MRI scan was opened and found to be filled with very thick mucus. The seventh-eighth cranial nerve complex was identified and both nerves appeared to be completely involved with a neoplastic process enlarging the nerves and encasing them. Intraoperative stimulation of the facial nerve at the brainstem did not result in any electromyographic response consistent with the patient's known complete left facial weakness, and an intraoperative auditory brainstem response could not be obtained. A preliminary pathology of the biopsy material from the abnormality along the seventh-eighth cranial nerve complex was consistent with metastatic adenocarcinoma. The posterior lip of the IAC was removed with the high speed drill and the entire lesion was resected including the involved left facial/vestibulocochlear nerve complex.

The immediate postoperative course was uncomplicated. Her preoperative deficits remained unchanged. The final pathology confirmed metastatic mucinous adenocarcinoma, with staining compatible with pulmonary or gastrointestinal/pancreatobiliary origin (Figure 3). It was felt likely the lesion arose in the GE junction with possible metastasis to the lungs and brain. On the day of discharge, she was ambulating with assistance and tolerating oral intake. She and her family ultimately declined additional investigation and treatment and she died on September 24th, 2013.

\section{Discussion}

The majority of mucinous adenocarcinomas is derived from simple glandular epithelia and originates from the gastrointestinal tract, and less frequently from breast, lung and pancreas or ovaries. The most common sites of distant metastasis of mucinous adenocarcinomas are ovary, peritoneum, liver or lung [1,2]. In many patients with unknown primary cancer, the brain metastasis could be the initial presentation [3-6]. According to Narita and Shibui, the primary sources of adenocarcinoma metastasis to the brain are lung (52.3\%), breast $(8.9 \%)$, renal (5.4\%), rectum (5.2\%), gastric (5.2\%), and colon (4.1\%) [7].
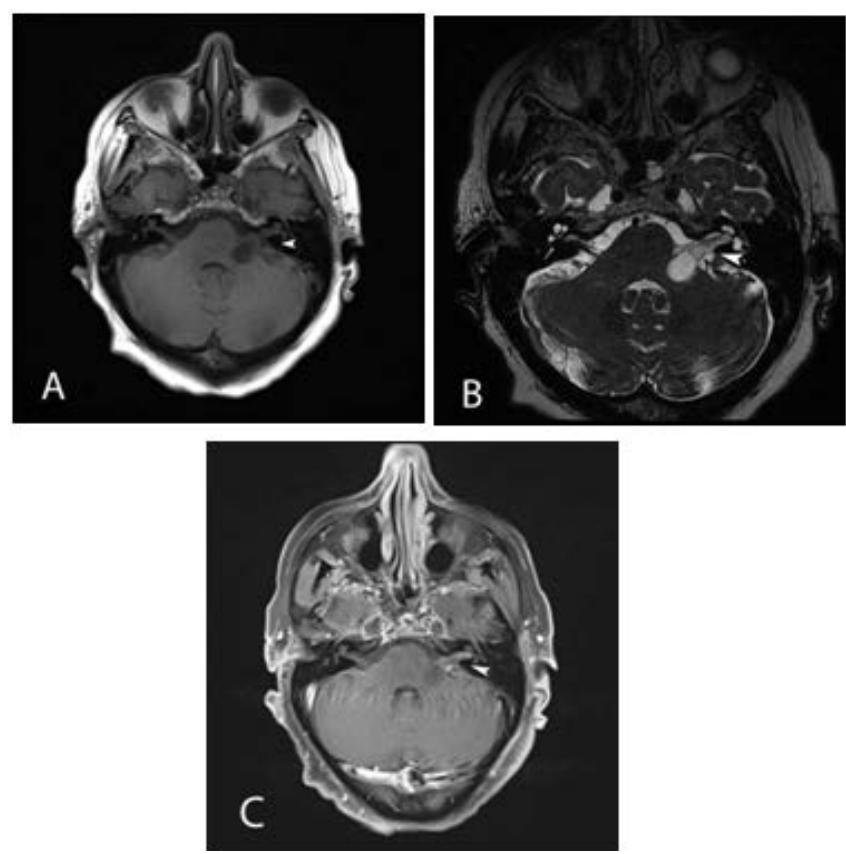

Figure 2: (A) Axial preoperative T1-weighted imaging without gadolinium demonstrates a mass lying within cerebellopontine angle (white arrowhead). (B) Axial T2-CISS image (high resolution T2-weighted imaging) of the cerebellopontine angle-internal auditory canal (CPA-IAC) cistern demonstrate the low signal mass as a filling defect in the high signal cerebrospinal fluid (CSF) of the CPA-IAC cistern (white arrowhead). (C) Axial T1-weighted imaging with gadolinium reveals a $1.2 \mathrm{~cm}$ enhancing cystic and solid lesion in the left cerebellar pontine angle extending into the left internal auditory canal which appears to involve cranial nerves VII and VIII (white arrowhead). 


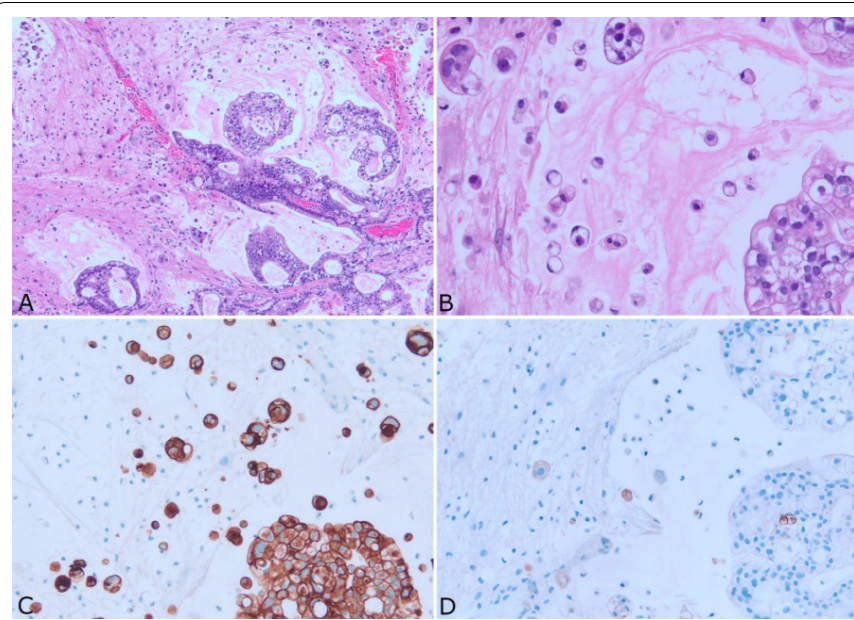

Figure 3: Metastatic mucinous adenocarcinoma with well-formed glands (A) immersed in s mucinous background Cells with signet ring appearance with pale cytoplasm and eccentric nuclei, filled with mucin are present $(B) A \& B H \& E$ respectively $\times 100 \& \times 400$ Cells are strongly positive for cytokeratin 7 (C) and only focally and weakly with cytokeratin 20 (D) (both x200). The morphology and immunophenotype are compatible with a variety of primary sites including upper GI, pancreata-biliary tract and lung. PCEA \& CDX2 showed very focal positivity, CDX2 being a marker of $\mathrm{Gl}$ origin, although it can be seen in other sites and Thyroid transcription factor 1 was negative (not shown).

Around $6 \%-10 \%$ of all intracranial tumors occur in the CPA $[8,9]$. In a series of $1354 \mathrm{CPA}$ tumors, $91.3 \%$ were vestibular schwannomas, $3.1 \%$ meningiomas, $2.4 \%$ epidermoids and about $3.2 \%$ of the tumors were trigeminal nerve schwannomas, arachnoid cysts, hemangiomas, hemangioblastoma, astrocytomas, medulloblastomas metastatic tumors, dermoids, lipomas, malignant teratomas, and chondrosarcomas [10]. Metastatic tumors to the CPA encompass about $0.7 \%$ of all tumors in this region, making this diagnosis extremely rare [9].

Clinical manifestations of a metastasis to the CPA are usually related to the regional cranial nerves with progressive hearing and balance dysfunction, facial weakness, and eventually dysphagia and dysphonia $[10,11]$. In the present report, we describe a unique case of mucinous adenocarcinoma metastatic to the facial/vestibulocochlear nerve complex which was radiographically occult for months until a repeat MRI scan revealed the lesion. Extensive systemic workup was likewise unrevealing prior to biopsy of the CPA lesion. A rapidly progressive clinical syndrome of the $\mathrm{CPA}$ in conjunction with an enhancing partially cystic mass on diagnostic imaging should include the differential diagnosis of a metastasis from a mucinous adenocarcinoma of undetected primary, even though it is extremely rare.

\section{References}

1. Chou YY, Jeng YM, Kao HL, Chen T, Mao TL, et al. (2003) Differentiation of ovarian mucinous carcinoma and metastatic colorectal adenocarcinoma by immunostaining with beta-catenin. Histopathol 43: 151-156.

2. Seidman JD, Elsayed AM, Sobin LH, Tavassoli FA (1993) Association of mucinous tumors of the ovary and appendix. A clinicopathologic study of 25 cases. Am J Surg Pathol 17: 22-34.

3. Agazzi S, Pampallona S, Pica A, Vernet O, Regli L, et al. (2004) The origin of brain metastases in patients with an undiagnosed primary tumour. Acta Neurochirurgica 146: 153-157.

4. D'Ambrosio AL, Agazzi S (2007) Prognosis in patients presenting with brain metastasis from an undiagnosed primary tumor. Neurosurg focus 22: E7.

5. Jenkinson MD, Haylock B, Shenoy A, Husband D, Javadpour M (2011) Management of cerebral metastasis: evidence-based approach for surgery, stereotactic radiosurgery and radiotherapy. Europ J Cancer 47: 649-655.

6. Ruda R, Borgognone M, Benech F, Vasario E, Soffietti R (2001) Brain metastases from unknown primary tumour: a prospective study. J Neurol 248 394-398.

7. Narita Y, Shibui S (2009) Strategy of surgery and radiation therapy for brain metastases. Int J Clin Oncol 14: 275-280.

8. Brunori A, Scarano P, Chiappetta F (1997) Non-acoustic neuroma tumor (NANT) of the cerebello-pontine angle: a 15-year experience. J Neurosurg Sci 41:159-168.

9. Moffat DA, Ballagh RH (1995) Rare tumours of the cerebellopontine angle. Clin oncol 7: 28-41.

10. Brackmann DE, Bartels LJ (1980) Rare tumors of the cerebellopontine angle. Otolaryngol Head Neck surg 88: 555-559.

11. Lalwani AK (1992) Meningiomas, epidermoids, and other nonacoustic tumors of the cerebellopontine angle. Otolaryngol Clin North Am 25: 707-728. 